\title{
Types of Statements on Emotion in Music
}

\author{
Benjamin Krämer
}

\begin{abstract}
A BSTRACT The question of emotion in music is addressed from a linguistic perspective, providing a typology of statements that can be made about that topic. In particular, it is analyzed how an interlocutor could react to such statements uttered by another person, and whether or how the content of the statements could be refuted by the listener, and possibly corroborated by the speaker. Furthermore, it is briefly discussed which theories of emotion in music are compatible with the respective types of statement and what illocutionary and perlocutionary function they may serve. KEYWORDS Emotion, music, linguistic analysis, typology
\end{abstract}

Music has often been said to be the language of emotion, ${ }^{1}$ but what does language have to say about emotion in music? Of course, the question of emotion in music has been treated extensively. However, I think that further clarification is possible on some points through the examination of statements on this very topic, i.e., statements on the relationship between music and emotions that could occur in everyday conversation or academic discourses. The relevance of this analysis is at least twofold. Firstly, it may be of intrinsic interest what people say about emotion in music: how can we express our experiences or judgments and convey them to others? Secondly, this analysis may complement a phenomenological and empirical perspective by approaching emotion in music via the indirect linguistic route. This does not mean that we should uncritically take any linguistic form to be a valid indicator of a phenomenon under analysis or an experience. However, by drawing on a typology of possible (and 'impossible') statements, one may use the different types of utterances to guide an introspective or empirical analysis of the experience represented by the linguistic forms: if a statement of a certain type can be made, what does it refer to, how can we grasp the essential properties of the phenomenon and its differentia specifica? Why can we make such statements and not others (that are nonsensical)? Conversely, a type of statement may be found to misguide a phenomenological or empirical analysis or to be at odds with a sound description of experiences or empirical facts. As a preliminary to an analysis along this route, I will discuss which types of statements and reactions are compatible with what kind of theory on emotion in music. A scholar can both put forth his or her own theory, and analyze which theories may be explicitly held or to which theories a speaker may be implicitly committed (as a 
precondition, a consequence, a corollary, and so forth) when making a statement on emotion and music. These are two different tasks, and I will switch between brief remarks of both kinds, according to what seems more interesting, appropriate and fruitful. My main interest, however, rests with the typology of statements.

The main questions of this essay can be posed thus: What, in general, may be said on (particular) emotions in (particular) music, i.e., what types of statements exist? What do such statements mean and imply? What are the criteria of their validity and their purpose? In particular, the analysis of these criteria and of the commitments implied in the statements is possible by noting how an interlocutor could react to statements uttered by another person, and whether and how the content of the statements could be refuted by the listener (as unjustified or nonsense), and possibly corroborated by the speaker.

I believe that each type of statement presented below cannot be reduced to another type, except if quite particular bridging theses are held. The typology is deduced from the simple distinction between the listener, the piece of music, and the artist or composer ${ }^{2}$ as the main point of reference of the statement: One may utter statements about the subjective impression of the listener as to the emotional appearance of the piece, the music referring symbolically to some emotion, and the artist's intention or the music as an indicator of his or her condition. This 'attribution' of emotion to different entities related to music is an essential aspect of a speaker's theory of emotion in music and therefore a typological criterion that is (certainly among others) suited for the analysis of statements. However, this does not mean that the types of statements are mutually exclusive or that they may be unequivocally mapped to distinct theories. They are simply more plausible in one theoretical framework than in another.

\section{I}

The first category of statements consists in what I shall term statements about impressions or appearance. It is exemplified by utterances such as, 'This piece of music sounds sad (to me)', or '(In my view) this music is/seems/appears (to be) sad'. The point of these statements is not to describe which emotions are caused by music, although the speaker may hold the theory that this amounts to the same thing: if music is or sounds sad, it makes people sad. ${ }^{3}$ This type of statement on effects I will only consider very briefly. It has been argued that listening to music can cause different emotions than those that are expressed or recognized in it; e.g., 
one can express delight that a work conveys an exemplary impression of a melancholic atmosphere; or it may not cause any emotion at all, as in the case of a completely analytic mode of listening. ${ }^{4}$ Conversely, I do not think that the main reason for calling a piece of music sad is that it causes sadness in the listener. ${ }^{5}$ For the present purpose, I will simply refer to the following statement as an illustration: 'How wonderfully sad that music is! It makes me melancholic, and I like that.' I think that the burden of proof is with those who argue that causation and impression amount to the same, despite the fact that there are two quite different types of statements. Furthermore, they should be able to explain how the view that impression and causation amount to the same thing could also apply to other phenomena or what accounts for the difference between emotions and other phenomena where this is not the case (e.g., consider the case of a work of art that is described as 'cold').

We shall now consider the possible answers to a statement such as, 'This piece of music sounds sad (to me)'. Then imagine a respondent saying something such as, 'No, it doesn't'. Whether and how this makes sense depends on what exactly the second speaker wishes to deny. It would not be adequate in most cases to respond by saying only, 'No, it doesn't', although there are at least four types of cases where a respondent might wish to reject the original speaker's statement on his impression of sadness. These types are as follows: The respondent disagrees as to the variety of emotion; the respondent thinks that the speaker is insincere; the respondent thinks that reasonable or experienced listeners should come to another conclusion; or the respondent thinks the speaker has used inadequate language.

1. The respondent may disagree as to the variety of emotion with a response such as, 'No, to me it sounds calm'. How is this possible? First, it has to be made clear how one can say of a piece of music that it is sad. $\mathrm{Mu}$ sic seems to belong to some kind of entity to which we often intuitively and naturally apply the word 'sad'. ${ }^{6}$ Indeed, we perceive a musical performance or a musical work as a distinct entity, as something belonging together and being separated from other phenomena, with some properties that characterize it as a whole, just as some other sound or noise: a shrill voice (heard without seeing the speaker, thus in particular perceived as a distinct entity), the strepitous creak of a door, and so on. ${ }^{7}$ If one is trying to listen to music while the neighbor has pumped up the volume of his stereo, we discern two entities, i.e., two musical events that can be characterized independently. Sometimes we may also wish to characterize only a part of a piece, for example, one passage or movement may be 
perceived as sad and another as joyful; the piano part in Beethoven's 4th piano concerto may be considered tender and imploring, while the unison strings seem grim and threatening. ${ }^{8}$ The choice of a level at which an entity is delimited and described in emotional terms is made implicitly or explicitly by the listener.

Having noted that music is perceived as an entity with certain properties or appearances, and leaving aside the task of defining music (we may simply say that listeners apply the word 'sad' to something they would call music), we may turn to the concept of emotion insofar as it is relevant to the emotional character ascribed to music in the present type of statement.

Emotions are phenomena that consist in several aspects or components, such as subjective feelings, arousal and valence, behavioral tendencies, facial and gestural expressions, cognitive contents and typical antecedents, and so forth. Depending on the theory of emotion one holds, different aspects are counted as part of the definition of emotion, while others are seen as causes, signs and consequences. Notwithstanding this possible vagueness of definition, cognitive schemata exist that serve to identify emotions even in the absence of the complete range of components. ${ }^{9}$ We could say in Wittgensteinian terms that we have learned the word 'sad' by being referred to some of these components. We have learned it implicitly (How do I know that this piece is sad? - It would be an answer to say: 'I have learned English'10).

Accordingly, something is called sad if it shows one or more of the typical aspects of sadness (outward signs and antecedents or introspective properties - introspection by a sad person, not by the beholder) or some appearance that suggests an analogy or similarity to such an aspect: slow movements, low arousal, a monotonous and low voice, references to causes such as a loss or loneliness, and so forth. This view has been defended in 'contour' or 'similarity' theories of musical expression. ${ }^{11}$ We say that a face or physiognomy (even of an animal which is not sad, such as a Saint Bernard) looks sad, that a posture or voice may be called sad. A desert landscape may also be said to look sad (the clue or link to the prototype being emptiness, loneliness or the 'cold' light), as may a painting with pale or dark colors, static composition, the portrayal of empty spaces, or portraying lonely or sad persons and situations that go along with sadness. We also sometimes use expressions indicating that there is sadness in something ('There was a lot of sadness in his face'). In the case of music, we may say that there is sadness, and that this sadness is in the music.

What components of sadness must be present or represented by anal- 
ogy, similarity, symbolism, or some other means, to cause listeners (with their different predispositions) to describe a specific piece of music as sad is an empirical rather than an analytical or phenomenological problem. ${ }^{12}$ Therefore, I will not speculate on this question, but assume that impressions of a piece's emotional character are phenomenally transparent - i.e., they are directly given to us (like an object is blue or green); the impression's content is consciously available, but one cannot determine introspectively what characterizes it as a mental fact per se (what different impressions have in common and what 'having' a certain impression amounts to), and what causes it. ${ }^{13}$ Thus, a person can make only speculative statements about the properties of the music that actually lead to the impression (in that person herself of himself) of sadness in a certain case, while the underlying mechanisms and criteria of this ascription are not accessible to introspection. The impression as such is neither subject to any doubt by the person (we do not 'know' about them, with all the qualifications and modalities that are applicable to knowledge, although the impression's content may be that something appears ambiguous to us), nor can it be proven or falsified by any external criteria. One has the impression and one cannot be mistaken about it, and another person does not have any means to argue about the impression itself; that is, the second person could not say, 'No, you are wrong, it does not appear sad to you', or 'No, your impression only appears to be one of sadness, but in fact it is one of joy'. What makes the type of statement considered here true is that the person has an identifiable impression and she feels that it is adequately subsumed under what is habitually called 'sadness', that she has recognized sadness, something known to her, and labeled it correctly.

Still, there are many opportunities for disagreement centered on statements about such an impression, as will be shown subsequently. In this case however, if the respondent disagrees as to the variety of emotion, he does not refute the original statement (that the speaker has the impression of sadness). He only rejects it in the sense that it does not reflect his own impression: that he disagrees; and he cannot, after consideration of or explication by the original speaker, adopt the first speaker's view or even imagine that it can be held (see below for a discussion of the possibility of such a change of mind).

A precondition for phenomenal transparency in the case of medially transmitted experiences (in the broadest sense, including live performances of music) is the transparency of the medium, i.e., the phenomenon that we are familiar with the technical modalities and the formal conventions of a medium to such a degree that we can interpret a representation 
in that medium directly; that some impression or meaning immediately and automatically comes to our mind without concentrating on its encoding. ${ }^{14}$ For example, when reading a text in one's own native language as opposed to one in a foreign writing system, we most often understand it in such way that we do not concentrate on the shape of the letters, often disregard typing errors or even the word choice and style unless we deliberately concentrate on it or unless some cue directs our attention towards these levels of the medium. Likewise, a westerner (or another person sufficiently familiar with western music) often easily recognizes some emotion in a pop song or a classical work without considering its structural features, whereas it may be difficult or impossible for her to identify any emotion in, say, the singing in a piece of Noh drama, even if she carefully follows its structure. She might say that this music 'doesn't tell her anything' - and, interestingly, that, e.g., if the music seems sad to her, this may be only because she is not familiar with the style of the piece, which 'may express something different, or any emotion at all' (see below on statements about expression). The opacity of the medium is one possible reason for a case where one person does not have a clear emotional impression at all. This probably occurs most often with early, atonal or foreign music, but can happen with any work. With regard to phenomenal transparency, it has to be noted that an unclear impression is possible (e.g., doubts about its character or the correct terms to state them), but doubts about having an impression or not are excluded. It is, e.g., impossible to doubt that one has doubts about the emotion one hears. A speaker or respondent can surely use terms that denote uncertainty about emotion in music, such as: 'I believe/suppose/guess that the music is somehow/probably/maybe sad', or: 'You seem to be unsure whether this music you described was really sad'. It is hard to find an example of a (according to my view, nonsensical) statement where the impression as such rather than its content is questioned, as we tend to relate any marks of doubt to the content, but it may be considered strange to say, 'You seem unsure whether you [now] have the impression that the music is probably/maybe/certainly sad/emotionally expressive/emotionally ambiguous' ('now' is added because one may be unable to exactly remember earlier impressions). To use another example, one would probably not say, 'You seem to be unsure whether you are unsure about the emotional character of the piece'.

Some persons may also be led to imagine a 'musical persona', i.e., a fictitious mind or person who experiences the emotion identified by the listener, while others may not; this may depend on the work. ${ }^{15}$ Possibly, 
a solo voice of any instrument is more readily associated with a person than a tutti and fortissimo that may be rather imagined as some impersonal force or architecture that is also perhaps associated with some emotion. This imagination can be assumed to underlie the same principle of phenomenal transparency and non-falsifiability as the ascription of an emotion as such.

It is evident that not every possible aspect of sadness can be found or represented by analogy or symbolism in a piece of music. Arousal, movement and valence are most easily represented, while the possible causes or antecedents and cognitive contents of sadness can hardly be conveyed without additional programmatic statements or titles. This explains why some authors have interpreted emotions expressed (and also caused) by music, or ascribed to music, as pseudo-emotions or otherwise 'unreal', 'incomplete' or 'etiolated'. ${ }^{16}$ I do not consider this distinction between real and unreal emotions all that helpful; all emotions ascribed to objects other than humans are 'pseudo-emotions' and all outer signs of emotions displayed by humans can obfuscate their real feelings. Emotions could be characterized as unreal if someone is mistaken about the cognitive appraisal of certain facts, or upset by certain fictional representations, and so on. Unless the concept of the 'realness' of an emotion is clarified, it is not very informative to use this term.

With the different degrees of relationship between the appearance of a phenomenon described as 'sad' and the appearance or sensations of a real person feeling sadness, statements on the 'sadness' of the phenomenon can also be called either figurative or literal speech (or maybe something on a continuum between). ${ }^{17}$ Today, and in western cultures in particular, many persons tend to assume an intimate and direct relationship between music and emotion, and they would readily apply emotional labels to it. This could explain why some deny that using emotional terms to describe music is metaphorical or metonymical speech, while others strongly defend this thesis. Consequently, a respondent may not only disagree as to the variety of emotion (e.g., calm instead of sad), but also reply

- that this particular piece, or any piece at all, does not really leave in her (personally) an impression of real emotionality, or

- that this piece, or any piece, does not really leave such an impression in anyone, and that such an 'impressionist' description of this or of any music is nonsense, fictitious, metaphorical, or a shorthand term for another kind of statement (such as on effects, symbolism, or expressiveness). 
Assuming that both interlocutors accept the idea that music can be characterized in emotional terms (being/sounding/appearing [to be] sad), based on such cues as described above, different persons may come to different conclusions as to how some music sounds emotionally.

As the range of components of an emotion is not completely present or represented in a specific piece or passage of music, an emotional impression can be ambiguous, and may somehow seem 'incomplete'. ${ }^{18}$ This is perhaps another reason why some have described emotions in music as, for example, 'not full-fledged' or as pseudo-emotions. However, for a given set of components, there is often one prototypical emotion that may differ according to personal dispositions but that is most readily recognized in the music. In such cases the listener would not call his impression 'incomplete', but would say rather that he really hears this emotion (that is readily recognized, but possibly 'incomplete' or ambiguous from an analytical perspective) as such in the music and that when he says that he does so, his statement is true. For example, we may speak of sadness in cases where low arousal and a negative valence are represented although there are many other emotional terms and schemata that match these criteria, and although the cognitive content of sadness (a loss or absence of a beloved person or object, helplessness, social exclusion, and so on) is completely absent. We may as well speak of melancholy, lassitude, disheartenment, depressiveness, grief, sorrow, or tristesse, as these emotions also match a less energetic and aversive state (although sometimes with some positive meta-emotional evaluations, such as the appreciation of melancholy). In some cases we may apply these terms to verbalize more differentiated impressions. The same probably holds for joy or joyfulness as prototypical in relation to similar emotions.

To acknowledge this probably inevitable ambiguity and incompleteness of the range of emotions heard in music and, at the same time, to recognize the tendency to ascribe prototypical emotions, are perhaps more illuminative and precise than to state that some emotions are more easily recognized and expressed in music than others. At least, both of these views may be complementary.

1*. A respondent may also express his differing impression somewhat more tentatively, as a suggestion or guess: 'Couldn't this also be calm rather than sad?' Due to the 'incompleteness' of emotion in music, it is possible for two listeners to at first disagree on the emotional character of a piece and for one of them to convince the other of his judgment (but not to 'convince' him in the sense of a logical argumentation), or for them to convince each another that their respective impressions are somehow 
conceivable or justifiable. The hearer of a statement about an emotional expression can be guided or motivated to agree with an impression different from hers, i.e., to 'hear the sadness' instead of some other emotion, by being referred to some traits of the music (i.e., to some alleged prototypical components), but she cannot be convinced in a strict sense - in the way that one can be convinced by some arguments that, e.g., the harmonic progression of a piece is the same as that of another work. In a certain way she can agree with the speaker that one can call the music sad, or even that it now seems completely evident to her that the music is indeed sad rather than anything else, or she may insist, 'This music still sounds calm to me'.

Changes in one's impressions - in particular, changes influenced by following a hint given by others or by concentrating on certain features of the music - can be subsumed under the concept of cognitive decentration: the ability or mental operation by which one can perceive and consider a phenomenon from more than one perspective and with regard to different sets of properties or with more than one conceptual scheme, to interpret it as something or something else, and to switch more or less deliberately between the perspectives as one's attention is directed willingly or unwillingly to different cues that suggest their adoption or their coordination and integration. ${ }^{19}$ Compare this to the drawing of the duckrabbit, an ambiguous figure in which we switch between seeing a rabbit and a duck. ${ }^{20}$ Like the contemplator of that drawing, we hesitate between the language of the description of immediate perceptions, of identification and certainty (this is a rabbit/an image of a rabbit, we see a rabbit, or it is sad music, it sounds sad), the language of 'objective' description (this drawing is made in such and such way that makes us see ..., the music has properties that lead us to the impression of sadness) - two levels of descriptive speech - and the language of subjectivity, interpretation, difference, intentionality, mere appearance, even illusion (we only see this as.../ this can be seen both as...[depending on...]; the music seems sad [to me, personally], we can try to hear it as sad and calm, I cannot hear it as sad [even if I try], and so forth). Thus, despite the phenomenal transparency of the impression under discussion, we can first recognize one emotion and regard this judgment as perfectly clear and immediately given (and thus make true statements about this impression), and then be lead to regard another impression as equally or more plausible and directly evident, in consequence of the utterances of others, the deliberate attempt to consider possible alternatives, or other means (and make equally true statements about this new impression). Furthermore, an existing impression 
can become clearer and more perspicuous if the listener pays attention to a work's formal characteristics. But these attempts may fail, so one may say, 'I still cannot hear the sadness that you say you can hear in this piece'. What we cannot do is try to hear what we already hear.

It is also possible to shift one's attention away from the direct impression of emotionality and concentrate on the presence or absence of its components in a work (otherwise, the above-mentioned explanations or theories and the terminology of 'unrealness' and 'incompleteness' would not be possible) or on the range of features of the work that may be assumed to cause the aspects of the impression by analogy, resemblance, and so forth, or on any other formal structures or properties of the work or the performance (e.g., by changing the mode of listening from 'impressionistic' to 'analytic').

Consequently, as the present type of statement on impressions does not have a truth condition that is strictly related to external criteria, we may now turn to another criterion by which a statement on an impression can be questioned, namely sincerity.

2. A respondent may think that the speaker is insincere when uttering a statement about his reputed impression. The respondent could say something like, 'No, it does not sound sad to you', but this seems quite unnatural. One would more likely answer that the speaker is only pretending to be sensitive and boasting about his differentiated impressions to, say, 'wow' someone, while he is really completely unmusical and unempathic or unfamiliar with the style of the work. Hence, even though one cannot be mistaken about the emotional appearance or impression of a piece to oneself, one could well deceive others about the existence or the content of such an impression.

3. A respondent may think that every reasonable or experienced listener should come to another conclusion, one that is the same as his own impression. She might say, 'The piece doesn't sound sad, it is calm, period!' or some less apodictic answer that goes in the same direction. Here, the speaker's force of judgment is questioned and the respondent holds the theory that the 'right' emotionality of a work can or will be 'correctly' grasped by all competent listeners in the same way without any room for personal impressions. A statement of the present type is true if all competent listeners have a recognizable impression and they all feel that it is adequately subsumed under what is habitually called 'sadness'. According to this assumption, the statement, 'The piece sounds sad' only makes sense without adding 'to me', as the piece sounds sad to everyone, or should sound so. The respondent may, in some extreme cases, even 
presume some distortion of perception or pathological sensation in the speaker. For example, if someone called the chorus in Beethoven's 9th symphony 'anxious', the reaction may be to express doubts about the personality of the speaker.

Here, a statement on an impression may converge with a strictly descriptive statement in the sense that, if everyone recognizes the same emotion, this can be attributed to the music in a very determinate way. Consequently, the respondent can regard an impression - even his own at an earlier point in time - as false in the sense that it is inadequate to the real character of the work. However, that impression itself, at the moment of its emergence and presence, is still phenomenally transparent: It is impossible to say that one wrongly thought that one considered the music as sad.

4. A respondent may think the speaker has used inadequate language. He may say something like, "No, "sad" is probably not the right word for what you want to say about this music'. Again, such an answer would probably not be reduced to the utterance, 'No, it doesn't [sound sad]!' but again, there may be occasions where this is almost conceivable. For example, if someone said, 'This music sounds schadenfroh' (probably being unaware of the meaning of this loanword), one could answer: 'No, it certainly doesn't'. The speaker is neither mistaken about his impression (which, again, we have argued that he cannot be) or the possibility of an impression, nor does he try to deceive someone, but he is mistaken about the conventional and understandable use of words for emotions and their rules of application to perceptible phenomena. He has not learned them, or the respective language, correctly, and uses the wrong word for what he means in describing the emotion he hears in the music.

\section{II}

The second type of statement may be said to be about symbolism, such as, 'This motif, consisting of slow descending triplets, symbolizes/expresses/ refers to sadness'. The concept of the symbol (or whatever the speaker may call the semiotic relation at hand) implied in such a statement may be either that of a purely conventional and arbitrary sign or of a relationship of similitude (comparable to iconic signs) or analogy.

As a reaction to this type of statement, an interlocutor can question the presence or correct identification of the signifier: there are no triplets, there are many major chords, and so on. To settle their dispute, they can take such actions as looking at the score, or listening to a recording more attentively. 
Another potential point of criticism with regard to this type of statement is to doubt that the symbolic relationship is stated correctly. For example, one may argue that triplets usually do not symbolize sadness. This kind of disagreement is cleared up with more difficulty, but the disputants may either refer to a larger body of musical pieces with their respective interpretations, information on musical conventions, styles of composition, and so forth, or they may try to make the formal resemblance more evident if they believe that this type of symbolism is at hand.

Still another reaction is to doubt that the music symbolizes anything at all, either in general or with regard to the specific piece - the position generally known as formalism. ${ }^{21}$

In sum, this type of statement is true if the elements or structures of a piece of music are, by some convention, similitude or analogy, related to an emotion, depending on the correct identification of the rule of symbolization.

If a speaker claims that the symbols he (in his mind, correctly) identifies are the cause, or as he may see it, the reason or motivation for his impression of sadness, he may identify a certain convergence between the two types of statements. He may link them by pointing to his faculty of musical judgment and possibly questioning that of others: He is able to intuitively grasp the emotional symbolism of the piece and his mode of listening is holistic, as analytic and intuitive judgments converge. However, this does not prevent others from holding that the music sounds different to them, e.g., calm instead of sad, even if they agree that the formal characteristics are present, or even if they concede that these features conventionally express sadness, but not in the present case. Thus, their personal impression may or may not be overruled by formal analysis.

Thus, the second type of statement is about semiotic relationships and symbolic conventions or 'iconic' symbols. This type can only be reduced to the first type if one holds a theory of symbolization that equates signification with the intended or quasi-universal arousal of impressions (i.e., ' $a$ means $b$ if $a$ evokes [the impression/feeling/idea, etc. of] $b$ '), unlike a theory that, e.g., defines symbolization as a recognizable rule-governed relationship of reference.

\section{III}

The third type of statement is about expression, symptoms, indications or intentions. For example, someone may claim, 'In the second movement of the $n$-th symphony the composer expresses his unrequited love to $X^{\prime}(1)$; 
'In this movement, the composer wants to explore the shades of sadness' (2) - a statement about an intention; or 'the composition is an emblematic expression of the fin-de-siècle sentiment of melancholy' (3) - a statement about the music as an indication or a symptom, or an unconscious expression of an emotion.

This type of statement involves the postulate of psychological (or biographical, or sociological, or some other type of) facts or mental states involving emotions and being external to the work or musical event itself; also, in particular, it involves an inference. According to different theories held by the speaker, which may not be mutually exclusive, music may directly express real emotions experienced by the composer, convey some emotions unconsciously, or be about emotions, i.e., characterize them in terms of typical and non-typical but possible developments over a period of time, their dynamic qualities, combinations of emotions to be found in two persons being confronted with each other, and so forth. ${ }^{22}$

Possible reactions to these types of statement include doubt as to the correctness of the inference with regard to its content (i.e., as in the previous cases, the variety of emotion), and to the underlying theory of expressiveness in general. A respondent can even hold that music is not suitable to express emotion, that composers in general do not aim at the expression of emotions, that one cannot make inferences as to their emotions or some general sentimental atmosphere, situation, or zeitgeist from their music, and so forth. This position parallels those reactions described in the case of the previous types of statements where the respondent denies that music has anything to do with emotions at all, or that, at least, the relationship is not of the kind implied in the respective type of statement. Thus the present type of statement is true if the right inferences are made (using adequate rules that link musical forms to states in the composer or his environment).

Statements on impressions and expression (and possibly on symbolism) converge if one believes he has intuitively comprehended the composer's intention, emotional state or other circumstances, and correctly assumed a causal relationship between the way the emotion is expressed in the piece and one's impression: The composer was sad, he used such and such a form to symbolize this, and the listener immediately identified this feeling, calling the piece sad.

If the speaker holds the theory that emotion is expressed in music if and only if a composer intentionally and knowingly encodes distinct emotions (his own, or the concept of the emotion as such) into clear-cut formal structures, statements of the present type either converge with 
those of the second, i.e., with statements about symbolism, as in the cases of (1) and (2), or they are nonsensical, as in the case of (3).

\section{IV}

After considering three types of statements with their referential and predicative aspects, their criteria of validity, and some possibilities to refute them, I will conclude by revisiting the question of what the purpose - the illocutionary and perlocutionary function - of such statements is. We have already seen that statements of the first, the 'impressionistic' type can be interpreted as expressive (of the speaker's impression) or as descriptive (of the work's appearance, or of the fact that it causes some impression or illusion). The strictly expressive function of such statements may be to voice one's experiences when listening to music and to share or compare them with that of others. This can serve to strengthen a sense of community or have a distinctive function with regard to musical, emotional and linguistic competence, in particular when it comes to 'right' and 'wrong' impressions and the use of differentiated emotional terms. Noting the emotional character of a piece can also serve as a starting point to a more structural analysis (e.g., asking what it is that makes the music sound sad).

Adding statements of the second, semiotic type can be seen as an attempt towards a more holistic appreciation of the work and to overcome merely subjective impressions, if one holds the theory that this is possible. Statements on symbolism may also serve as justifications or motives to adopt a certain perspective with regard to impressions or appearance (cf. above on cognitive decentration).

The second type can also be the basis for generalizations as to the form of music in general, or of some style or genre, and its emotional idioms: What does it mean, in general, to use a certain structure? How is it used and which emotions does it refer to (which, according to a theory of meaning as use, amounts to the same)?

If someone announces that she will attempt to give a 'hermeneutic analysis' or 'interpretation' of a musical work, she may grosso modo be expected, among other things, to utter statements of the second or third type (dealing with symbolism and expression), or both, depending on the understanding of the method. Statements on the impressions of the speaker or of others will be considered mostly irrelevant as strict arguments for a hermeneutic theory, although they may fulfill different illustrative, heuristic or informative functions in analyses of musical works. Sometimes they also occur as more or less disguised statements of another type: 'This 
passage sounds/is sad', which then means that it expresses sadness, or that its structure refers to sadness. Statements of the second and third type are also sometimes driven by interest in the composer rather than in a work: his style, his personality, his method of composing, or his conception of a faithful performance, for example, or in some group of composers, a school or a stylistic movement. Finally, all three kinds of statements can be used to reflect upon performances (one's own or others) and the relationship between the emotions of performers and those emotions 'in' the work (in the above-mentioned different senses).

Statements of the third, inferential type, could also be intended as an attempt of some kind of reductionism - a work is only a symptom of $x$ - which is sometimes also meant as a form of criticism: The work is not 'pure', its aesthetic is not disinterested, its production is driven by ephemeral affects or sentimental fashions, and so forth. Of course, the alternative of an anti-reductionist message is also possible: the work is more than a symptom of $x$, it is the product of an independent genius with his particular emotional experiences, a work that sublimates its affective material, and so on. Both of these lines of interpretation can be refuted as the fallacy of inferring a work's aesthetic value from the circumstances of its production. However, the different types of statements also enter into the evaluation of a work in other regards, e.g., if the speaker judges the appropriateness and originality or the effectiveness with which an emotional impression is created in the listener.

Considering the pragmatic aspect of the types of statements under analysis, we may finally note that some persons rarely talk about emotions, in particular with regard to music (whether they listen to it in a sentimental mode or not), and that to some persons, the above statements may indicate an overestimation of the relationship between music and emotion, an inflationary use of emotional terms in the analysis and criticism of music and everyday talk about it, and a loss of alternative aesthetic and analytic approaches. Both groups may then hold the view that those statements are, if not senseless, then useless or, to some extent, superfluous.

\section{Notes}

1. For a discussion of this assumption, see Stephen Davies, 'Is Music a Language of Emotions?', British Journal of Aesthetics 23 (1983), 222-223.

2. I will only refer to listening rather than to reading a score as the more pertinent case, while someone may possibly also call a piece of music 'sad' after 
a look at the score - this however remains debatable. Furthermore, I will use terminology that follows the concepts usually applied to instrumental classical music: composer, work, performance, and so forth. I think my arguments can be easily adapted to other 'ontologies' of music - for an overview of such ontologies, see Andrew Kania, 'Pieces of Music: The Ontology of Classical, Rock, and Jazz Music' (PhD diss., University of Maryland, 2005).

3. Stanley Speck, "'Arousal Theory” Reconsidered', British Journal of Aesthetics 28 (1988), 40-47.

4. On exemplary impressions, see, e.g., R.T. Allen, 'The Arousal and Expression of Emotion by Music', British Journal of Aesthetics 30 (1990), 57-61. On 'aesthetic emotions' see Klaus R. Scherer, 'Which Emotions Can Be Induced by $\mathrm{Mu}-$ sic? What Are the Underlying Mechanisms? And How Can We Measure Them?' Journal of New Music Research 33 (2004), 239-251.

5. At least, it will not necessarily cause 'normal' sadness, i.e., sadness that is recognizable as such and expressed by statements such as, 'I am sad'. Listeners of 'sad' music will not necessarily say of themselves that they are sad, and they can be trusted at that point, because feeling sad (and, as a consequence, potentially being able to express this verbally - given the necessary linguistic competences) is one part (and an essential one in almost any definition) of sadness as a genuine and full-fledged emotion.

6. On the meaning of such statements, see Malcolm Budd, 'Music and the Communication of Emotion', Journal of Aesthetics and Art Criticism 47 (1989), 129-38, and V. A. Howard, 'On Musical Expression', British Journal of Aesthetics 11 (1971), 268-8o.

7. On music as such an entity, see Kendall Walton, 'What is Abstract about the Art of Music?' Journal of Aesthetics and Art Criticism 46 (1988), 351-64.

8. On the case of several voices, see Jenefer Robinson, "The Expression and Arousal of Emotion in Music', Journal of Aesthetics and Art Criticism, $5^{1}$ (1994), 13-22.

9. See e.g. Scherer, 'Which Emotions Can Be Induced by Music? What Are the Underlying Mechanisms? And How Can We Measure Them?' for a quite comprehensive definition of emotion as a multicomponent process and its application to music.

10. Ludwig Wittgenstein, Philosophical Investigations (Oxford: Blackwell, 2001), $\mathbb{3} 81$. The reader may notice that some of my premises are Wittgensteinian. As I cannot argue for or against them within the limits of this paper, I ask the reader to accept them at least for the sake of the argument.

11. Stephen Davies, 'The Expression of Emotion in Music', Mind 89 (1980), 268 80.

12. In this view, music is 'readily hearable as' sad; cf. Jerrold Levinson, 'Musical Expressiveness as Hearability-as-expression', in Contemporary Debates in 
Aesthetics and the Philosophy of Art, ed. Mathew S. Kieran (Oxford: Blackwell, 2006), 192-206.

13. On the notion of phenomenal transparency, see George E. Moore, 'The Refutation of Idealism', Mind 12 (1903), 433-453. I cannot discuss the diverse criticisms of the thesis of phenomenal transparence here, but simply assume that it can be defended using both phenomenological and linguistic arguments.

14. For an application to opera, see Peter Kivy, 'Speech, Song, and the Transparency of Medium: A Note on Operatic Metaphysics', Journal of Aesthetics and Art Criticism 52 (2004), 63-68.

15. For a critical view of theories of a musical persona, see Derek Matravers, 'The Experience of Emotion in Music', Journal of Aesthetics and Art Criticism 61 (2003), 353-363.

16. For a critical view of this 'pseudo-emotion' theory, see Peter Kivy, 'Feeling the Musical Emotions', British Journal of Aesthetics 39 (1999), 1-13.

17. Again, in a Wittgensteinian (and somewhat Salomonic) perspective, we may say that both analogies can be misleading in their respective ways. If we call statements on musical emotion 'figurative', we could be led to think that there could be a better, more direct way to speak about what we usually describe in emotional terms. According to this (debatable) intuition, we would only call some speech figurative (or metaphorical) if there is another way to describe the same phenomenon in a non-figurative way, even if those formulations are difficult to find. However, we would regard it as a loss of information, a change in the level of description, if emotional terms in a description of a piece of music were replaced by formal parameters of the piece or of some passages. Identifying the expression of sadness is not the same as identifying formal properties, and we would say that a hearer has missed something if she is unable to identify some emotion expressed by a piece. If we call a statement about musical expression a 'literal' use of emotion words, we will be led to ask whose emotion it is that we speak of, as we only attribute 'real' emotions to humans; and we have to acknowledge that those statements we usually apply to emotions cannot be applied, as a whole, to emotions said to be expressed by music (e.g., music cannot pretend to be sad, and a person would also miss something if he tried to learn emotion words solely by listening to music others have described with these words). See Davies, 'Is Music a Language of Emotions?'

18. E.g., lacking clear cognitive content that is typical for an emotion, such as a loss in the case of grief. For a discussion of such arguments on the lack of cognitive content, see, e.g., Robinson, 'The Expression and Arousal of Emotion in Music'.

19. Jean Piaget, Psychologie de l'intelligence (Paris: Armand Collin, 1967), 100 and 190-191. 
20. Wittgenstein, Philosophical Investigations, $\int \mathbb{\int 1 6 5 - 7 0 . ~}$

21. This view is often attributed to Eduard Hanslick, Vom Musikalisch-Schönen: Ein Beitrag zur Revision der Ästhetik der Tonkunst (Leipzig: Weigel, 1854), but Hanslick himself does not always follow his own formalist argument, as has been shown by Peter Kivy, 'Something I've Always Wanted to Know about Hanslick', Journal of Aesthetics and Art Criticism 46 (1988), 413-17.

22. On some of these theories, see Robinson, 'The Expression and Arousal of Emotion in Music'. 